(c) 2021 Universidad Nacional Autónoma de México, Facultad de Estudios Superiores Zaragoza.

Este es un artículo Open Access bajo la licencia CC BY-NC-ND (http://creativecommons.org/licenses/by-nc-nd/4.0/).

TIP Revista Especializada en Ciencias Químico-Biológicas, 24: 1-9, 2021.

https://doi.org/10.22201/fesz.23958723e.2021.393

\title{
Las galectinas en la coagulación sanguínea
}

\author{
Berenice Fernández-Rojas', Rodrigo Arreola-Díaz ${ }^{2}$, Pedro Antonio Hernández-Cruz ${ }^{1}$, \\ Itandehui Belem Gallegos-Velasco ${ }^{1}$, Carlos Josué Solórzano-Mata ${ }^{3}$, \\ Liliana Argueta-Figueroa ${ }^{4}$ y Jesús Hernández-Juárez ${ }^{4 *}$ \\ ${ }^{1}$ Laboratorio de Glicobiología, Genómica y Proteómica del Cáncer, Centro de Investigación Facultad de Medicina \\ UNAM-UABJO, ${ }^{3}$ Laboratorio de Bioquímica de Proteínas y Glicopatologías, Facultad de Odontología, Centro \\ de Investigación UNAM-UABJO, Facultad de Medicina y Cirugía, Universidad Autónoma "Benito Juárez" \\ de Oaxaca. Ex-Hacienda de Aguilera S/N Carretera a San Felipe del Agua, 68020, Oaxaca de Juárez, México. \\ ${ }^{2}$ Departamento de Inmunología, Escuela Nacional de Ciencias Biológicas, Instituto Politécnico Nacional. \\ Prolongación de Carpio y Calle Plan de Ayala s/n, Santo Tomás, Alcaldía Miguel Hidalgo, 11340, Ciudad de \\ México, México. ${ }^{4}$ CONACYT-Facultad de Odontología, Universidad Autónoma "Benito Juárez" de Oaxaca, Av. \\ Universidad S/N, Colonia Cinco Señores, 68120 Oaxaca de Juárez, México. E-mail: *jhdz0912@gmail.com.
}

\section{RESUMEN}

La hemostasia es un fenómeno de alto valor biológico que comprende la participación de elementos celulares y plasmáticos a través de los que se forman coágulos en los sitios de lesión vascular para evitar que las personas mueran a causa de una hemorragia. Por el contrario, en la trombosis, se forma un coágulo patológico o trombo que obstruye el flujo sanguíneo afectando a tejidos y órganos, lo que puede llevar a la muerte.

Por su parte, las galectinas son un grupo de proteínas de la familia de las lectinas, que, no poseen actividad enzimática ni tienen un origen inmune, pero reconocen a carbohidratos específicos en las células. Se ha demostrado su contribución en procesos fisiológicos y patológicos, como el cáncer y la aterosclerosis. En esta revisión se describirá, el papel de la galectina-1 (Gal-1), la galectina-3 (Gal-3) y la galectina-8 (Gal-8) en el proceso hemostático, así como los hallazgos más recientes que sugieren su probable participación en las trombosis.

Palabras clave: hemostasia, galectinas, trombosis.

\section{Galectins in blood coagulation}

\begin{abstract}
Haemostasis is a phenomenon of high biological value that involves the participation of cellular and plasma elements through which clots are formed at site of vascular injury to prevent people die by a hemorrhage. On the contrary, in thrombosis, a pathological clot or thrombus is formed that obstructs blood flow and can lead patients to death.

On the other hand, galectins are a group of proteins belonging to lectins families, which do not possess enzymatic activity or have an immune origin, but recognize specific carbohydrates in cells. Its contribution has been determined in physiological and pathological processes, such as cancer and atherosclerosis. In this review the role of galectin-1 (Gal-1), galectin-3 (Gal-3) and galectin-8 (Gal-8) in the hemostatic process will be described, as well as the most recent findings that suggest their probable involvement in thrombotic diseases.
\end{abstract}

Keywords: haemostasis, galectins, thrombosis.

Artículo recibido el 20 de marzo del 2021.

Artículo aceptado el 02 de diciembre del 2021. 


\section{INTRODUCCIÓN}

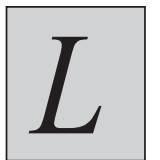

a coagulación sanguínea normal ocurre cuando de manera ordenada y equilibrada la hemostasia detiene la hemorragia en los sitios de daño vascular. Sin embargo, es posible que la formación de coágulos se presente bajo diferentes circunstancias y en tiempos equivocados, obstruyendo el flujo sanguíneo y causando isquemia en los tejidos. A este estado patológico se le denomina trombosis, que es un proceso poligénico en el que participan factores hereditarios y adquiridos. Los factores de riesgo hereditarios son poco frecuentes, pero no así los factores adquiridos. Es el caso de la diabetes mellitus que eleva el riesgo de aterotrombosis en los pacientes debido a la disfunción endotelial y a las alteraciones que promueven la hipercoagulabilidad de la sangre (Fattah, Shaheen \& Mahfouz, 2003; Peterson \& Hart, 2016). Esta enfermedad es un ejemplo de cómo los carbohidratos pueden modificar el curso normal del sistema de coagulación orillándolo hacia la trombosis; sin embargo, los receptores de los carbohidratos también ejercen sus efectos sobre este sistema. En esta revisión describimos algunos aspectos de la participación de las proteínas en la coagulación sanguínea, específicamente en el papel que tienen las galectinas en las diferentes etapas de la hemostasia, así como su contribución en la trombosis. Las galectinas son un tipo especial de proteínas llamadas lectinas, que reconocen a carbohidratos específicos en las células y que han mostrado tener participación en diferentes procesos biológicos, incluyendo el metabolismo y la inflamación (Johannes, Jacob \& Leffler, 2018), sin embargo, en la actualidad se conoce poco sobre su participación en la hemostasia y la trombosis.

\section{Galectinas}

El término lectina se aplica a proteínas o glicoproteínas, sin actividad enzimática, que no tienen un origen inmune y reconocen de manera específica a los carbohidratos de la membrana celular o en el citoplasma aglutinan células y precipitan a los glicoconjugados (Van den Steen, Rudd, Dwek \& Opdenakker, 1998).

Las galectinas son una familia de proteínas conservadas a través de la evolución que pueden descifrar glicocódigos específicos en macromoléculas complejas situadas en las membranas celulares o en la matriz extracelular, a través de un dominio de alrededor de 130 aminoácidos denominado dominio de reconocimiento de carbohidratos (DRC), con el que reconocen a los $\beta$-galactósidos (Rabinovich, 1999; Kasai \& Hirabayashi, 1996). Las galectinas están involucradas en fenómenos de inmunomodulación (Giovannone, Smith, Treanor \& Dimitroff, 2018) y regulación del metabolismo (Brinchmann, Patel \& Iversen, 2018), la aterosclerosis (Agnello et al., 2017), el desarrollo, la progresión y metástasis del cáncer (Chou, Chen, Kuo \& Sytwu, 2018), así como también participan en la biología de las alteraciones hematológicas como el mieloma múltiple y las leucemias (Ruvolo, 2019; Storti, Marchica \& Giuliani,
2017). Ejercen sus efectos biológicos a través del reconocimiento de azúcares específicos en ligandos intracelulares, receptores de membrana y glicoproteínas extracelulares, y sus diferentes funciones dependen de su localización celular.

Las galectinas pueden tener efectos contrarios entre ellas, así, la Gal-1 inhibe la desgranulación de mastocitos y la migración de los leucocitos, mientras que, la Gal-3 promueve la desgranulación de los mastocitos y migración leucocitaria. En mamíferos, las galectinas se clasifican en tres tipos con base en la organización de su dominio y son numeradas siguiendo el orden de su descubrimiento. El primero corresponde a las galectinas proto o prototipo, que, usualmente forman homodímeros, donde cada subunidad contiene 1 DRC. Este tipo incluye a la Gal-1, $-2,-5,-7,-10,-11,-13,-14$ y -15 . La Gal-5 es la única de este tipo que no forma dímeros. El segundo tipo corresponde a las galectinas de repetición en tándem, en las cuales $2 \mathrm{DRC}$ distintos se unen mediante un péptido de enlace de longitud variable. Este tipo se compone de la Gal-4, -6, -8, -9 y -12 y suelen ser monoméricas. Por su parte, la Gal-3, es la única galectina tipo quimera, que posee en su extremo C-terminal el DRC unido a una cola polipeptídica N-terminal rica en residuos de prolina y glicina, con la que forma oligómeros (Vasta, Feng, Bianchet, Bachvaroff \& Tasumi, 2015; Dings, Miller, Griffin \& Mayo, 2018). Las galectinas $-5,-6,-11$ y -15 no se encuentran en humanos. La Figura 1 muestra la clasificación de la galectinas en los mamíferos.

Las galectinas se expresan en diferentes tejidos, algunas tienen una distribución amplia y otras restringida. De manera especial para esta revisión son las galectinas que se expresan en células sanguíneas, plaquetas y en células endoteliales, destacando la Gal-1, -3 y -8 (Brinchmann, Patel \& Iversen, 2018), las que participan en la inflamación, pero también en la hemostasia.

\section{Hemostasia y Trombosis}

Las células endoteliales mantienen la sangre líquida dentro de los vasos sanguíneos a través del equilibrio fisiológico entre los factores procoagulantes, anticoagulantes y fibrinolíticos. El endotelio es el componente principal de este sistema que en condiciones normales posee propiedades antiplaquetarias, anticoagulantes y fibrinolíticas (Krüger-Genge, Blocki, Franke \& Jung, 2019); sin embargo, cuando es lesionado o activado, pierde sus propiedades propiciando que el equilibrio antes mencionado se pierda y se desplace hacia los factores procoagulantes. La hemostasia comprende tres etapas: la vasoconstricción, la activación plaquetaria (compuesta por la adhesión y agregación plaquetaria) y la fase plasmática, que en conjunto y de manera coordinada detienen la hemorragia tras una lesión vascular. La vasoconstricción disminuye el flujo de sangre en los sitios de daño vascular al minimizar la pérdida hemática y facilitar la adhesión y agregación de las plaquetas sobre las que se desarrollará la fase plasmática de la hemostasia. Esta última fase involucra la activación en cascada de los factores de coagulación y culmina 


\begin{tabular}{|c|c|c|c|}
\hline Tipo & Proto & $\begin{array}{l}\text { Repetición en } \\
\text { tándem }\end{array}$ & Quimera \\
\hline DRC & 1 & 2 & 1 \\
\hline \multicolumn{4}{|l|}{ Monómero } \\
\hline \multicolumn{4}{|l|}{$\begin{array}{l}\text { Dímero u } \\
\text { oligómero }\end{array}$} \\
\hline Galectinas & $\begin{array}{c}-1,-2,-5,-7 \\
-10,-11,-13 \\
-14 \text { y }-15\end{array}$ & $\begin{array}{l}-4,-6,-8 \\
-9 y-12\end{array}$ & -3 \\
\hline
\end{tabular}

Figura 1. Clasificación de las galectinas en mamíferos. Se clasifican en tres tipos: (a) las galectinas proto, que poseen 1 dominio de reconocimiento de los carbohidratos (DRC), forman homodímeros, cada subunidad con 1 DRC, a excepción de la Gal-5 que es monomérica; (b) las galectinas de repetición en tándem poseen 2 DRC distintos y unidos por un fragmento peptídico; (c) en las galectinas quimera, el monómero posee $1 \mathrm{DRC}$ en su extremo C-terminal unido a una cola polipeptídica $\mathrm{N}$-terminal con secuencias repetitivas de glicina y prolina, con la que puede formar un oligómero.

cuando la trombina convierte el fibrinógeno en fibrina(Hoffman \& Monroe, 2001). El proceso de coagulación de la sangre es estrictamente regulado por los anticoagulantes naturales, como el sistema de la proteína $\mathrm{C}$ activada.

La fibrinólisis por su parte tiene la función de degradar el coágulo de fibrina una vez que la hemostasia cumplió con su objetivo, es un proceso enzimático que al igual que la hemostasia comprende la participación de moléculas activadoras, como el activador tisular del plasminógeno (t-PA), y moléculas inhibidoras, entre estas, el inhibidor del activador tisular del plasminógeno tipo 1 (PAI-1) y la $\alpha-2$ antiplasmina (Chapin \& Hajjar, 2015). Tras la degradación del coágulo y la reparación tisular se reestablece el balance entre los factores procoagulantes, anticoagulantes y fibrinolíticos.

En la trombosis también se forman coágulos, sin embargo, siempre son patológicos, reciben el nombre de trombos y pueden ocasionar la muerte. Los trombos se forman en vasos sanguíneos o cavidades cardiacas. La trombosis ocurre cuando un trombo obstruye el flujo local de la sangre en venas o arterias provocando que los tejidos y células irrigados por estos vasos afecten a cualquier órgano del cuerpo. Existen tres situaciones que predisponen a la trombosis: las alteraciones en la pared vascular, la estasis sanguínea y las alteraciones procoagulantes de la sangre. Por otra parte, a toda situación que eleve el riesgo de trombosis se le denomina trombofilia, y puede ser hereditaria o adquirida, primaria o secundaria, aguda y crónica (Majluf-Cruz \& Espinosa-Larrañaga, 2007). Por ejemplo, una trombofilia primaria es la deficiencia de la proteína C, el aumento en la actividad del factor VIII (FVIII) o de PAI-1. En cualquiera de estos casos, el desbalance entre los factores procoagulantes, anticoagulantes y proteínas de la fibrinólisis conduce a un estado trombofílico. En cambio, una trombofilia secundaria es aquella que no tiene su origen en el sistema de coagulación pero que indirectamente eleva el riesgo trombótico, como ocurre en el cáncer, el síndrome de anticuerpos antifosfolípidos y la diabetes, entre otras, cada una de ellas afecta de manera crónica al sistema de coagulación. En otras situaciones como en los traumatismos, las quimioterapias o la coagulación intravascular diseminada, el daño al sistema de coagulación es agudo (Majluf-Cruz \& Espinosa-Larrañaga, 2007; Majluf-Cruz, 2017).

La Figura 2, muestra de manera sencilla el equilibrio fisiológico entre la hemostasia, la fibrinólisis y sus mecanismos de regulación natural, y bajo qué situaciones el riesgo trombótico se eleva.

\section{EL PAPEL DE LAS GALECTINAS EN LA HEMOSTASIA}

La participación de las galectinas en la hemostasia se ha documentado en los últimos 15 años, demostrándose su relación con los principales componentes del sistema de coagulación: las plaquetas, los factores de coagulación y las células endoteliales (Tabla I).

En las plaquetas, la Gal-1 y la Gal-8 favorecen el desarrollo de procesos fundamentales de la hemostasia primaria, como son la adhesión y la agregación de las plaquetas, así como el incremento de los niveles intracelulares de calcio, la liberación 


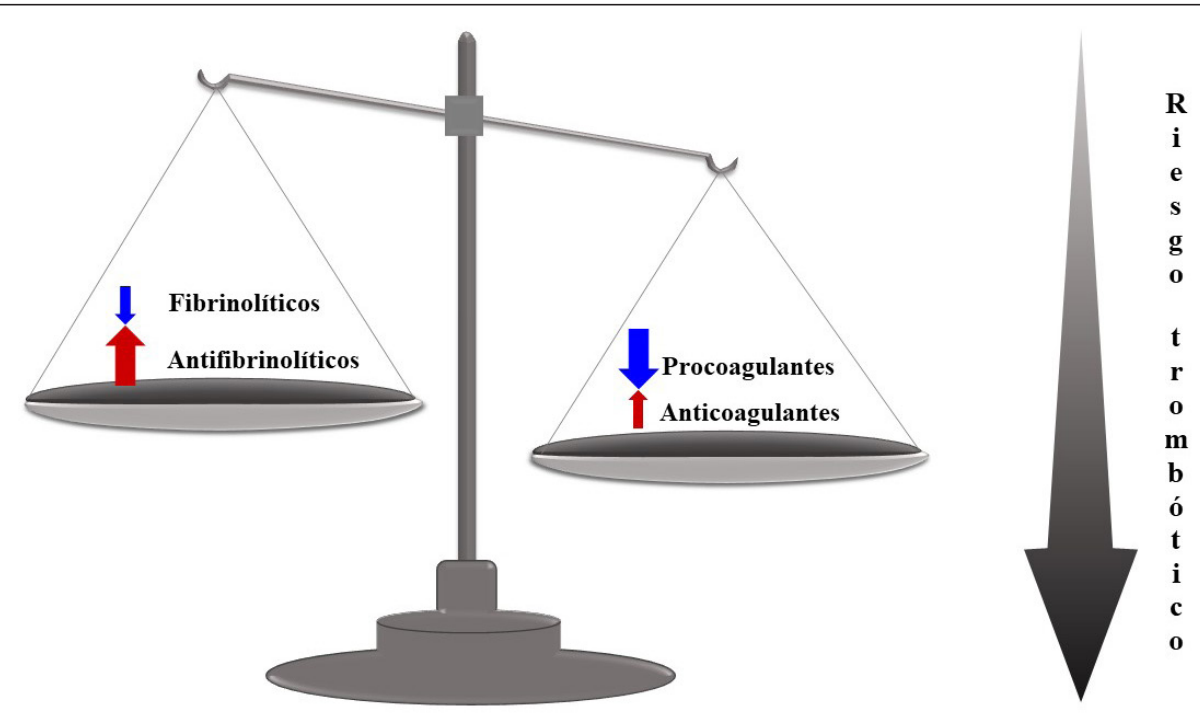

Figura 2. La coagulación sanguínea. En condiciones fisiológicas la sangre se mantiene líquida dentro de los vasos sanguíneos y se torna sólida únicamente en los sitios de daño vascular. Lo anterior es posible debido al equilibrio entre los factores procoagulantes y anticoagulantes de la hemostasia. Por otro lado, la fibrinólisis en equilibrio con sus inhibidores naturales degradará al coágulo evitando que se aloje en vasos de diámetro menor. Sin embargo, existen situaciones que favorecen la formación desregulada de los coágulos, mejor conocidos como trombos que pueden obstruir por completo la luz de los vasos sanguíneos. La actividad incrementada de las proteínas antifibrinolíticas y disminución de la actividad de los anticoagulantes naturales favorece por un lado que la hemostasia forme coágulos cuándo y dónde no se requieren, y por otro, que la fibrinólisis no sea capaz de degradarlos. Ambas situaciones elevan el riesgo de trombosis en las personas, poniendo en riesgo su salud y su vida.

de micropartículas y los cambios morfológicos que son característicos de una plaqueta activada (Romaniuk et al., 2010; Romaniuk et al., 2012; Pacienza et al., 2008). La Gal-8 favorece la generación de tromboxano A2, secreción de P-selectina y la desgranulación plaquetaria. Se ha demostrado que las glicoproteínas $\alpha \mathrm{IIb}$ y Ib-V actúan como los posibles receptores de la Gal-8 en las plaquetas. La interacción entre esta lectina y la glicoproteína Ib desencadena la vía de señalización Src/PLC2 $\gamma /$ ERK y PI3K/Akt (Romaniuk, 2010), mientras que su unión a la glicoproteína IIb, desencadena la fosforilación de la integrina $\beta 3$, Syk, MAPKs, PI3K y PLC $\gamma 2$, además de la liberación del tromboxano A2 y la movilización del calcio (Romaniuk et al., 2012). En ambos casos, las vías de señalización mediadas por la Gal-8 tienen como desenlace la activación de las plaquetas. Sin embargo, uno de los hallazgos de mayor relevancia fue demostrar que la Gal-8 se expone en la membrana de las plaquetas por efecto de la trombina, con su participación en la hemostasia primaria y probablemente, en la trombosis (Romaniuk et al., 2010; Romaniuk et al., 2012). De manera similar a la Gal-8, la Gal-1 actúa sobre las plaquetas mostrando un efecto sinérgico con los agonistas adenosín difosfato (ADP) y trombina, entre sus efectos más importantes están los cambios que ejerce a nivel del citoesqueleto, en la conformación de la glicoproteína IIb/IIIa y su participación en los mecanismos de liberación de las micropartículas (Pacienza et al., 2008). En conjunto, los hallazgos antes mencionados sugieren que la Gal-1 y la Gal-8 actúan como agonistas plaquetarios.
Por otro lado, resultados in vitro y en animales deficientes de la Gal-1 y la Gal-3 demostraron que ambas lectinas regulan la interacción del factor von Willebrand (VWF) con las plaquetas (Saint-Lu et al., 2012). Se encontró que la deficiencia de las galectinas favorece la interacción VWF-plaquetas, además de que la velocidad de formación de trombos en los animales de experimentación fue más rápida al emplear un modelo experimental de trombosis.

En la fase plasmática, reaparece la participación de la Gal8. Esta galectina permite la endocitosis del factor $\mathrm{V}$ en los megacariocitos (Zappelli, van der Zwaan, Thijssen-Timmer, Mertens \& Meijer, 2012; Gertz \& Bouchard, 2015), que son las células precursoras de las plaquetas. Este evento es crucial para la hemostasia, ya que el factor $\mathrm{V}$ será liberado posteriormente por las plaquetas en la fase inicial de la hemostasia secundaria y servirá como cofactor del factor $\mathrm{X}$ durante la activación de la protrombina a trombina, estaúltima, la enzima más importante de la fase plasmática de la hemostasia. En adición, se ha demostrado que el FVIII circula en complejo con el VWF, pero también con la Gal-1 y la Gal-3 (O'Sullivan et al., 2016), destacando que la Gal-1 regula la actividad del FVIII. Debido a la importancia clínica que representa este factor en medicina, el entendimiento de los mecanismos biológicos de la Gal-1 en la regulación de la actividad de este factor plasmático podría aportar información relevante no solo en el ámbito de la trombosis, sino de la hemofilia, principalmente, en el desarrollo de los inhibidores, 
Tabla I. Participación de las galectinas en la hemostasia y la trombosis.

\begin{tabular}{|c|c|c|}
\hline $\begin{array}{c}\text { Gal-1 } \\
(1 \mathrm{DRC})\end{array}$ & $\begin{array}{c}\text { Gal-3 } \\
(1 \mathrm{DRC})\end{array}$ & $\begin{array}{c}\text { Gal-8 } \\
(2 \text { DRC) }\end{array}$ \\
\hline $\begin{array}{l}\text { Agonista plaquetario (Romaniuk et al., } \\
\text { 2010; Romaniuk et al., 2012; Pacienza } \\
\text { et al., 2008) }\end{array}$ & $\begin{array}{l}\text { Regula la interacción VWF- } \\
\text { plaquetas (Saint-Lu et al., 2012) }\end{array}$ & $\begin{array}{l}\text { Se expresa en la membrana de las plaquetas por } \\
\text { efecto de la trombina (Romaniuk et al., 2010; } \\
\text { Romaniuk et al., 2012) }\end{array}$ \\
\hline $\begin{array}{l}\text { Favorece la adhesión y agregación } \\
\text { plaquetaria (Romaniuk et al., 2010; } \\
\text { Romaniuk et al., 2012; Pacienza et al., } \\
\text { 2008) }\end{array}$ & $\begin{array}{l}\text { Forma un complejo con el FVIII } \\
\text { (O'Sullivan et al., 2016) }\end{array}$ & $\begin{array}{l}\text { Agonista plaquetario (Romaniuk et al., 2010; } \\
\text { Romaniuk et al., 2012; Pacienza et al., 2008) }\end{array}$ \\
\hline $\begin{array}{l}\text { Regula la interacción VWF-plaqueta } \\
\text { (Saint-Lu et al., 2012) }\end{array}$ & $\begin{array}{l}\text { Su deficiencia causa un estado } \\
\text { procoagulante, contribuye a la } \\
\text { patogénesis de la diabetes mellitus } \\
\text { y a la vasculopatía (Darrow \& } \\
\text { Shohet, 2015) }\end{array}$ & $\begin{array}{l}\text { Favorece la adhesión y agregación plaquetaria } \\
\text { (Romaniuk et al., 2010; Romaniuk et al., 2012; } \\
\text { Pacienza et al., 2008) }\end{array}$ \\
\hline $\begin{array}{l}\text { Inhibe la actividad del FVIII } \\
\text { (O’Sullivan et al., 2016) (Roda et al., } \\
\text { 2009; Nagaoka } t \text { al., 2002) }\end{array}$ & $\begin{array}{l}\text { Causa disfunción endotelial (Ou } \\
\text { et al., 2019) }\end{array}$ & $\begin{array}{l}\text { Generación de tromboxano, secreción de P-selectiva } \\
\text { y desgranulación plaquetaria } \\
\text { (Romaniuk et al., 2010; Romaniuk et al.,2012) }\end{array}$ \\
\hline $\begin{array}{l}\text { Actúa en el sistema de fibrinólisis, } \\
\text { incrementa moderadamente la actividad } \\
\text { de t-PA y favorece la degradación de la } \\
\text { malla de fibrina (Roda et al., 2009) }\end{array}$ & $\begin{array}{l}\text { Biomarcador de aterosclerosis } \\
\text { (Oyenuga et al., 2019) }\end{array}$ & $\begin{array}{l}\text { Incrementa los niveles intracelulares de } \mathrm{Ca}^{2+} \text {, } \\
\text { liberación de micropartículas y cambios } \\
\text { morfológicos de la plaqueta activada (Romaniuk et } \\
\text { al., 2010; Romaniuk et al., 2012; Pacienza et al., } \\
\text { 2008) }\end{array}$ \\
\hline $\begin{array}{l}\text { Forma un complejo con el FVIII y } \\
\text { regula su actividad (O'Sullivan et al., } \\
\text { 2016) }\end{array}$ & & $\begin{array}{l}\text { Promueve la interacción entre leucocitos, células } \\
\text { endoteliales y plaquetas (Romaniuk et al., 2010; } \\
\text { Romaniuk et al., 2012) }\end{array}$ \\
\hline \multirow{3}{*}{$\begin{array}{l}\text { Actúa como un anticoagulante y como } \\
\text { fibrinolítico }\end{array}$} & & Se desconoce su papel en la trombosis \\
\hline & & $\begin{array}{l}\text { Sus posibles receptores son glicoproteínas } \alpha \mathrm{IIb} \text { y } \\
\mathrm{Ib}-\mathrm{V} \text { (REF) }\end{array}$ \\
\hline & & $\begin{array}{l}\text { Efecto sinérgico con agonistas de ADP y trombina, } \\
\text { cambios en el citoesqueleto y conformacionales de } \\
\text { la glicoproteína IIb/IIIa (Pacienza et al., 2008). }\end{array}$ \\
\hline
\end{tabular}

La Tabla I muestra de manera sintetizada los resultados de los diferentes estudios citados en el texto sobre el probable papel de las galectinas en la hemostasia y la trombosis. Donde: Akt, proteína cinasa B; $\mathbf{C a}^{2+}$, ión calcio; ERK, cinasa regulada por señales extracelulares; FVIII, factor VIII; MAPKs, proteínas cinasas activadas por mitógenos; PI3K, fosfatidilinositol- 3-cinasa; PLC2 $\gamma$, fosfolipasa C gamma 2; Src, protooncogén tirosinaproteína cinasa; Syk, tirosinas cinasas Syk; VWF, Factor de von Willebrand. 
ya que es muy bien conocido que los carbohidratos son una pieza clave en la respuesta inmune contra el FVIII.

Quizás los hallazgos más importantes de las galectinas en el sistema de coagulación sean aquellos relacionados con el endotelio, ya que debemos recordar que son estas células las que dictan los caminos a seguir ante una lesión vascular o bien, cuando las células endoteliales son activadas. Resultados in vitro muestran que la Gal-8 activa al endotelio favoreciendo el aumento de la adhesión de las plaquetas, probablemente, debido al aumento de la expresión endotelial del VWF. Aunado a esto, la Gal-8 incrementa la expresión de las moléculas inflamatorias, como el ligando de quimiocina 1 , factor estimulante de colonias de granulocitos y macrófagos, interleucina 6 y el ligando de la quimiocina 5 , y en menor grado de la proteína quimiotáctica de los monocitos 1 , ligando de quimiocina 3 e interleucina 8 (Cattaneo et al., 2014). El estudio muestra que la Gal-8 promueve la interacción entre los leucocitos, las células endoteliales y las plaquetas, tal y como ocurre en la inflamación, sin embargo, la interacción entre estas células acontece también en el proceso hemostático, y en el peor de los escenarios, en la trombosis.
Por su parte, la Gal-1 actúa en el sistema de la fibrinólisis, se une al t-PA incrementando moderadamente su actividad catalítica (Roda et al., 2009). Ante esta evidencia se puede decir que la Gal-1 favorece la degradación de la malla de fibrina, pero lo más interesante radica en que esta lectina parece actuar como un anticoagulante al regular la actividad del FVIII y como un fibrinolítico al estimular la degradación de la fibrina, lo cual indiscutiblemente imprime un mayor valor biológico a esta proteína en términos del sistema de coagulación. Aunque algunos resultados muestran que la desregulación de la Gal-1 afecta la actividad de t-PA en distintos procesos celulares (Roda et al., 2009; Nagaoka, Strital, Kouyoumdjian \& Borges, 2002), se desconoce su implicación en las enfermedades trombóticas. La Figura 3 muestra de manera general el principal papel de las galectinas en la hemostasia.

\section{El papel de las galectinas en la trombosis}

Estudios in vitro, en animales y en humanos, han aportado evidencia sobre la participación de las galectinas en el desarrollo de la trombosis (Tabla I). Los antecedentes muestran que la Gal-3 juega un papel en la aterogénesis (Oyenuga, Folsom,

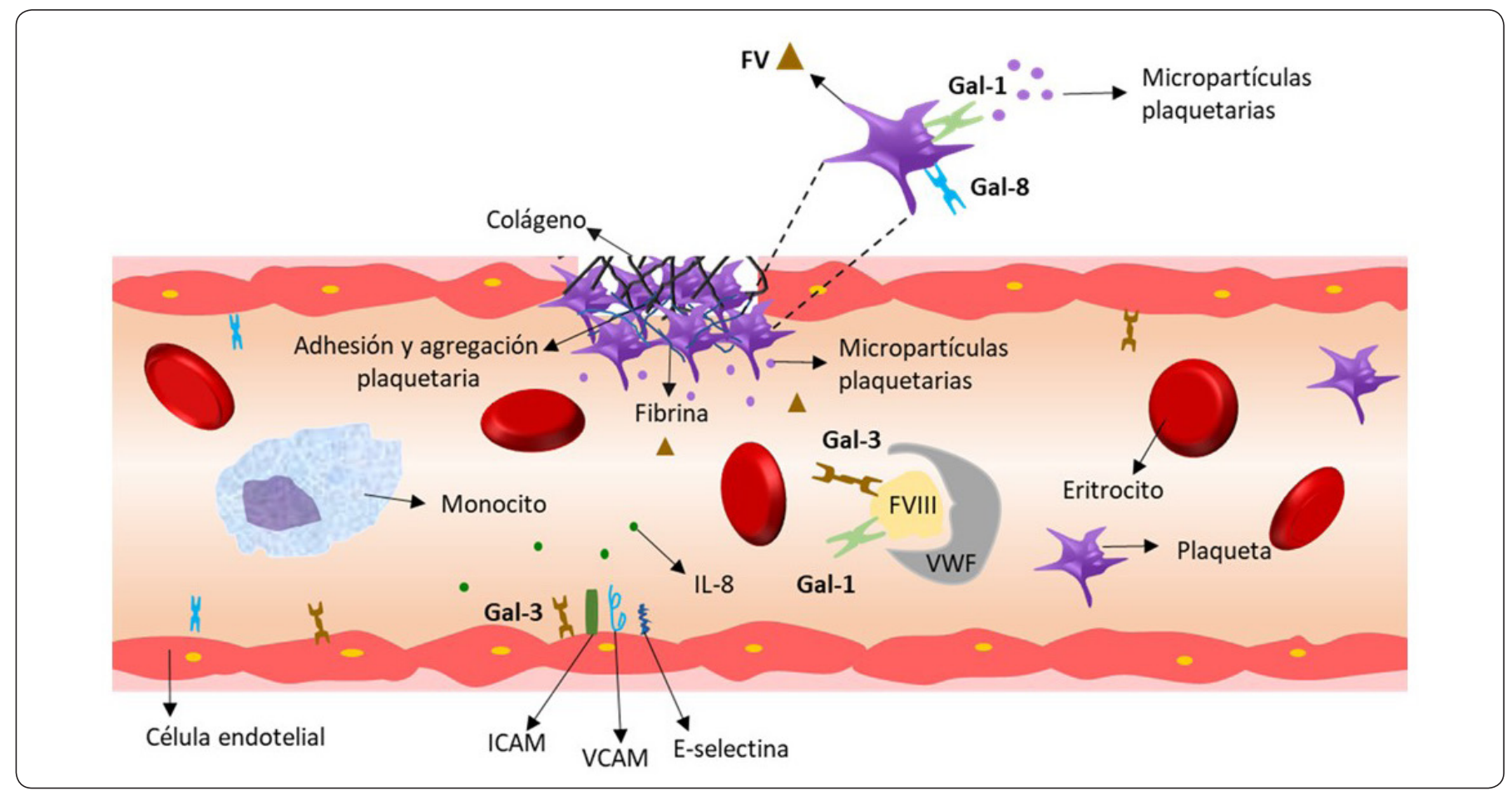

Figura 3. El papel de las galectinas en la hemostasia. La Gal-1 y Gal-8 interaccionan con receptores en la membrana de las plaquetas favoreciendo la adhesión y agregación plaquetaria. En adición, la Gal-1 promueve la liberación de micropartículas plaquetarias, mientras que la Gal-8, en una etapa temprana actúa sobre los megacariocitos permitiendo la endocitosis del FV que más adelante será liberado de los gránulos alfa de las plaquetas activadas. Hasta este punto, ambas galectinas ejercen su función sobre la hemostasia primaria y sobre la fase inicial de la hemostasia secundaría. Por otro lado, la Gal-1 y la Gal-3 viajan en circulación en complejo con el FVIII y el factor de von Willebrand (VWF), donde, la Gal-1, actúa regulando la actividad del FVIII. En un escenario diferente, la Gal-3 y la Gal-8 ejercen su acción sobre el endotelio causando cambios proinflamatorios. En el caso particular de la Gal-3, incrementa la expresión de moléculas de adhesión en las células endoteliales para el reclutamiento de leucocitos en la pared del vaso sanguíneo y posteriormente, ingresar al subendotelio y contribuir al proceso aterogénico. 
Fashanu,Aguilar \& Ballantyne, 2019), y con ello, en la trombosis arterial. Se han propuesto diferentes mecanismos, sin embargo, un estudio reciente muestra que la Gal-3 induce hacia una disfunción endotelial al aumentar los efectos de las lipoproteínas de baja densidad oxidadas en estas células (Ou et al., 2019). Sin embargo, su participación en la trombosis venosa también ha sido documentada. En animales de experimentación se observó que la Gal-3 muestra niveles plasmáticos elevados desde la etapa inicial de una trombosis venosa y que su expresión en la pared vascular correlaciona directamente con el tamaño del trombo (DeRoo et al., 2015). En el mismo estudio, también se observó que los niveles plasmáticos de Gal-3 se incrementan en pacientes con trombosis venosa (DeRoo et al., 2015). En especial este último hallazgo podría posicionar a la Gal-3 como un blanco terapéutico. Por otro lado, la contribución de la Gal-3 en la enfermedad tromboembólica venosa quedó demostrada al estudiar la asociación entre los niveles plasmáticos de esta lectina con la incidencia de la enfermedad trombótica en un estudio que evaluó los plasmas de 9, 916 pacientes. Los resultados revelaron la existencia de una asociación positiva entre los niveles plasmáticos de la lectina con la enfermedad trombótica (Fashanu et al., 2017), lo que significa que a mayor concentración plasmática de la Gal-3 el riesgo de trombosis venosa se incrementa. Pese a estos hallazgos, es importante mencionar que el comportamiento de la Gal-3 en la hemostasia juega un papel dual. Es decir, su deficiencia también modifica el curso normal del sistema de coagulación y de otros sistemas. Esto se demostró al evaluar ratones knockout para la Gal-3 y ratones control, ambos grupos fueron alimentados con una dieta alta en grasas o una dieta estándar. La dieta alta en grasas causó resistencia a la insulina. Los resultados demostraron que los ratones deficientes de la Gal-3 y con resistencia a la insulina presentaron una mayor hiperglucemia e intolerancia a la glucosa, así como niveles más disminuidos de insulina en comparación con los ratones control. De manera general, se observó que la deficiencia de la Gal-3 exacerba las alteraciones metabólicas de los ratones con resistencia a la insulina, además de que promueve a un estado procoagulante, ya que el tiempo de protrombina tiende a acortarse (Darrow \& Shohet, 2015). Los autores concluyeron, entre otras cosas, que la deficiencia de la Gal-3 contribuye a la patogénesis de la diabetes mellitus y a la vasculopatía asociada, por lo que, en concentraciones normales la Gal-3 posee un efecto protector contra la trombosis. A pesar de que otras galectinas tienen un papel en la hemostasia, su relación con la trombosis no ha sido explorada.

\section{Discusión}

La identificación de biomarcadores tempranos y el conocimiento de nuevos mecanismos implicados en la hemostasia y la trombosis son estrategias que se adoptan para intentar disminuir la incidencia de esta enfermedad, que cabe mencionar, es la primera causa de muerte en el mundo (Wendelboe \& Raskob, 2016), pero también, para diseñary ofrecer mejores tratamientos a los pacientes. En la mayoría de los casos, la trombosis se manifiesta como el evento final de otra enfermedad. Los mecanismos son diversos, sin embargo, la disfunción endotelial es quizás la causa principal que desequilibra al sistema de coagulación. En enfermedades como la diabetes mellitus, los pacientes padecen de disfunción endotelial pero también de estados de hipercoagulabilidad que en conjunto ocasionan que un porcentaje muy elevado de pacientes muera a causa de un infarto. Recientemente, un estudio reveló que, en adición a los factores de riesgo ya conocidos, los niveles séricos de la Gal-3 son un factor de riesgo independiente de la enfermedad cardiovascular en pacientes diabéticos (Tan etal.,2019). El papel de la Gal-3 en el endotelio es de gran magnitud y por esto ha sido propuesta como un biomarcador de la aterosclerosis (Oyenuga et al., 2019). Este conocimiento es de gran importancia no solo para los pacientes con diabetes, sino para otros individuos que padecen de enfermedades en las que la aterosclerosis llega a ser causa de muerte, como sucede con la periodontitis, que puede o no ser una comorbilidad en los pacientes diabéticos.

Al igual que la Gal-3, la Gal-8 podría tener un lugar especial en el campo de la trombosis. Si bien esta galectina muestra efectos proinflamatorios en el endotelio, se desconoce si proteínas anticoagulantes, antiagregantes y fibrinolíticas modifican su expresión. La expresión alterada de estas proteínas endoteliales, al igual que la cuantificación del óxido nítrico permitiría tener mayor evidencia de cómo la Gal-8 y quizá otras galectinas inducen a una trombosis. En otras palabras, los efectos de la Gal-8 en el endotelio conducirían a la aterosclerosis, pero, además, ocasionarían un desequilibrio entre la hemostasia y sus sistemas de regulación natural favoreciendo la aparición de estados procoagulantes. Lo mismo podría aplicar para la Gal-3 y explicar el por qué sus niveles plasmáticos están elevados en pacientes con trombosis venosa, en la cual, la aterosclerosis no tiene un papel trascendental, pero sí los estados de hipercoagulabilidad. Sin embargo, la Gal-8 no solo muestra efectos sobre el endotelio, sino que además es un agonista plaquetario, por lo que su evaluación en patologías donde las plaquetas son hiperactivas se convierte en un amplio campo de estudio. La Gal-1 es un ejemplo claro de la diversidad de efectos de estas proteínas en la hemostasia y de la necesidad de realizar más investigación al respecto, por un lado, la Gal-1 promueve la agregación plaquetaria y la liberación de micropartículas, pero, por otro, regula la actividad del VWF sobre las plaquetas, inhibe la actividad del FVIII en la circulación sanguínea y quizás favorezca la activad de t-PA. Pese a estos antecedentes su papel en la trombosis aún se desconoce.

\section{ConClusiones}

Las Gal-1, Gal-3 y Gal-8, son las galectinas que de una u otra forma mantienen una relación con el sistema de coagulación (Figura 2). La mayor parte de la evidencia se centra en cómo estás lectinas regulan la actividad de las plaquetas y sobre 
sus efectos nocivos en las células endoteliales. Mientras que poco o casi nada se sabe acerca de su participación con los componentes de la fase plasmática de la hemostasia. La Gal-3 es la más estudiada y de la que se tiene evidencia sobre su participación en la trombosis venosa y arterial, misma que ha sido propuesta como un biomarcador de la aterosclerosis. En cambio, se desconoce si las Gal-1 y Gal-8 mantienen alguna relación con las enfermedades trombóticas, lo cual, deja abierta la posibilidad de generar nuevas líneas de investigación.

\section{Agradecimientos}

Agradecemos la beca de Retención CONACYT otorgada a Berenice Fernández-Rojas.

\section{REFERENCIAS}

Agnello, L., Bivona, G., Lo Sasso, B., Scazzone, C., Bazan, V., Bellia, C. \& Ciaccio, M. (2017). Galectin-3 in acute coronary syndrome. Clinical biochemistry, 50(13-14), 797-803. https://doi.org/10.1016/j.clinbiochem.2017.04.018.

Brinchmann, M. F., Patel, D. M. \& Iversen, M. H. (2018). The Role of Galectins as Modulators of Metabolism and Inflammation. Mediators of inflammation, 2018, 9186940. https://doi.org/10.1155/2018/9186940.

Cattaneo, V., Tribulatti, M. V., Carabelli, J., Carestia, A., Schattner, M. \& Campetella, O. (2014). Galectin-8 elicits pro-inflammatory activities in the endothelium. Glycobiology, 24(10), 966-973. https://doi.org/10.1093/ glycob/cwu060.

Chapin, J. C. \& Hajjar, K.A. (2015). Fibrinolysis and the control of blood coagulation. Blood Reviews, 29(1), 17-24. https:// doi.org/10.1016/j.blre.2014.09.003.

Chou, F. C., Chen, H. Y., Kuo, C. C. \& Sytwu, H. K. (2018). Role of Galectins in Tumors and in Clinical Immunotherapy. International Journal of Molecular Sciences, 19(2), 430. https://doi.org/10.3390/ijms19020430.

Darrow, A. L. \& Shohet, R. V. (2015). Galectin-3 deficiency exacerbates hyperglycemia and the endothelial response to diabetes. Cardiovascular Diabetology, 14, 73. https:// doi.org/10.1186/s12933-015-0230-3.

DeRoo, E. P., Wrobleski, S. K., Shea, E. M., Al-Khalil, R. K., Hawley, A. E., Henke, P. K., Myers, D. D., Jr., Wakefield, T. W. \& Diaz, J. A. (2015). The role of galectin-3 and galectin-3-binding protein in venous thrombosis. Blood, 125(11), 1813-1821. https://doi.org/10.1182/ blood-2014-04-569939.

Dings, R., Miller, M. C., Griffin, R. J. \& Mayo, K. H. (2018). Galectins as Molecular Targets for Therapeutic Intervention. International Journal of Molecular Sciences, 19(3), 905. https://doi.org/10.3390/ijms19030905.

Fashanu, O. E., Heckbert, S. R., Aguilar, D., Jensen, P. N., Ballantyne, C. M., Basu, S., Hoogeveen, R. C., deFilippi, C., Cushman, M. \& Folsom, A. R. (2017). Galectin-3 and Venous Thromboembolism Incidence: the Atherosclerosis
Risk in Communities (ARIC) Study. Research and Practice in Thrombosis and Haemostasis, 1(2), 223-230. https://doi. org/10.1002/rth2.12038.

Fattah, M. A., Shaheen, M. H. \& Mahfouz, M. H. (2003). Disturbances of haemostasis in diabetes mellitus. Disease Markers, 19(6), 251-258. https://doi. org/10.1155/2004/797458.

Gertz, J. M. \& Bouchard, B. A. (2015). Mechanisms Regulating Acquisition of Platelet-Derived Factor V/Va by Megakaryocytes. Journal of Cellular Biochemistry, 116(10), 2121-2126. https://doi.org/10.1002/jcb.25163.

Giovannone, N., Smith, L. K., Treanor, B. \& Dimitroff, C. J. (2018). Galectin-Glycan Interactions as Regulators of B Cell Immunity. Frontiers in Immunology, 9, 2839. https:// doi.org/10.3389/fimmu.2018.02839.

Hoffman, M. \& Monroe, D. M., 3rd (2001). Acell-based model of hemostasis. Thrombosis and Haemostasis, 85(6), 958-965.

Johannes, L., Jacob, R. \& Leffler, H. (2018). Galectins at a glance. Journal of Cell Science, 131(9), jcs208884. https:// doi.org/10.1242/jcs.208884.

Kasai, K. \& Hirabayashi, J. (1996). Galectins: a family of animal lectins that decipher glycocodes. Journal of Biochemistry, 119(1), 1-8. https://doi.org/10.1093/oxfordjournals. jbchem.a021192.

Krüger-Genge, A., Blocki, A., Franke, R. P. \& Jung, F. (2019). Vascular EndothelialCell Biology:An Update. International Journal of Molecular Sciences, 20(18), 4411. https://doi. org/10.3390/ijms20184411.

Majluf-Cruz, A. (2017). Trombofilia [Thrombophilia]. Gaceta Médica de México, 153(4), 427-429. https://doi. org/10.24875/GMM.M17000013.

Majluf-Cruz,A., \&Espinosa-Larrañaga, F.(2007). Fisiopatología de la trombosis. Gaceta Médica de México, 143 (S1), pp.11-14.

Nagaoka, M. R., Strital, E., Kouyoumdjian, M. \& Borges, D. R. (2002). Participation of a galectin-dependent mechanism in the hepatic clearance of tissue-type plasminogen activator and plasma kallikrein. Thrombosis Research, 108(4), 257-262.https://doi.org/10.1016/s0049-3848(02)00393-6.

O’Sullivan, J. M., Jenkins, P. V., Rawley, O., Gegenbauer, K., Chion, A., Lavin, M., Byrne, B., O'Kennedy, R., Preston, R. J., Brophy, T. M. \& O’Donnell, J. S. (2016). Galectin-1 and Galectin-3 Constitute Novel-Binding Partners for Factor VIII. Arteriosclerosis, Thrombosis, and Vascular Biology, 36(5), 855-863. https://doi.org/10.1161/ ATVBAHA.115.306915.

Ou, H. C., Chou, W. C., Hung, C. H., Chu, P. M., Hsieh, P. L., Chan, S. H. \& Tsai, K. L. (2019). Galectin-3 aggravates ox-LDL-induced endothelial dysfunction through LOX-1 mediated signaling pathway. Environmental Toxicology, 34(7), 825-835. https://doi.org/10.1002/tox.22750.

Oyenuga, A., Folsom, A. R., Fashanu, O., Aguilar, D. \& Ballantyne, C. M. (2019). Plasma Galectin-3 and 
Sonographic Measures of Carotid Atherosclerosis in the Atherosclerosis Risk in Communities Study. Angiology, 70(1), 47-55. https://doi.org/10.1177/0003319718780772.

Pacienza, N., Pozner, R. G., Bianco, G. A., D’Atri, L. P., Croci, D. O., Negrotto, S., Malaver, E., Gómez, R. M., Rabinovich, G. A. \& Schattner, M. (2008). The immunoregulatory glycan-binding protein galectin-1 triggers human platelet activation. FASEB Journal: Official Publication of the Federation of American Societies for Experimental Biology, 22(4), 1113-1123. https://doi.org/10.1096/ fj.07-9524com.

Peterson, S. B. \& Hart, G. W. (2016). New insights: A role for O-GlcNAcylation in diabetic complications. Critical Reviews in Biochemistry and Molecular Biology, 51(3), 150-161.https://doi.org/10.3109/10409238.2015.1135102.

Rabinovich, G.A.(1999). Galectins: an evolutionarily conserved family of animal lectins with multifunctional properties; a trip from the gene to clinical therapy. Cell Death and Differentiation, 6(8), 711-721. https://doi.org/10.1038/ sj.cdd.4400535.

Roda, O., Ortiz-Zapater, E., Martínez-Bosch, N., GutiérrezGallego, R., Vila-Perelló, M., Ampurdanés, C., Gabius, H. J., André, S., Andreu, D., Real, F. X. \& Navarro, P. (2009). Galectin-1 is a novel functional receptor for tissue plasminogen activator in pancreatic cancer. Gastroenterology, 136(4), 1379-e5. https://doi. org/10.1053/j.gastro.2008.12.039.

Romaniuk, M. A., Croci, D. O., Lapponi, M. J., Tribulatti, M. V., Negrotto, S., Poirier, F., Campetella, O., Rabinovich, G. A. \& Schattner, M. (2012). Binding of galectin-1 to $\alpha \mathrm{IIb} \beta \square$ integrin triggers «outside-in» signals, stimulates platelet activation, and controls primary hemostasis. FASEB Journal: Official Publication of the Federation of American Societies for Experimental Biology, 26(7), 2788-2798. https://doi.org/10.1096/fj.11-197541.

Romaniuk, M. A., Tribulatti, M. V., Cattaneo, V., Lapponi, M. J., Molinas, F. C., Campetella, O. \& Schattner, M. (2010). Human platelets express and are activated by galectin- 8 . The Biochemical Journal, 432(3), 535-547.https:/doi.
org/10.1042/BJ20100538.

Ruvolo, P. P. (2019). Galectins as regulators of cell survival in the leukemia niche. Advances in Biological Regulation, 71, 41-54. https://doi.org/10.1016/j.jbior.2018.09.003.

Saint-Lu, N., Oortwijn, B. D., Pegon, J. N., Odouard, S., Christophe, O. D., de Groot, P. G., Denis, C. V. \& Lenting, P. J. (2012). Identification of galectin-1 and galectin-3 as novel partners for von Willebrand factor. Arteriosclerosis, Thrombosis, and Vascular Biology, 32(4), 894-901. https:// doi.org/10.1161/ATVBAHA.111.240309.

Storti, P., Marchica, V. \& Giuliani, N. (2017). Role of Galectins in Multiple Myeloma. International Journal of Molecular Sciences, 18(12), 2740. https://doi.org/10.3390/ ijms 18122740 .

Tan, K., Cheung, C. L., Lee, A., Lam, J., Wong, Y. \& Shiu, S. (2019). Galectin-3 and risk of cardiovascular events and all-cause mortality in type 2 diabetes. Diabetes/ Metabolism Research and Reviews, 35(2), e3093. https:// doi.org/10.1002/dmrr.3093.

Van den Steen, P.E., Rudd, P. M., Dwek, R. A. \& Opdenakker, G. (1998). Concepts and principles of O-linked glycosylation. Critical Reviews in Biochemistry and Molecular Biology, 33(3), 151-208. https://doi. org/10.1080/10409239891204198.

Vasta, G. R., Feng, C., Bianchet, M. A., Bachvaroff, T. R. \& Tasumi, S. (2015). Structural, functional, and evolutionary aspects of galectins in aquatic mollusks: From a sweet tooth to the Trojan horse. Fish \& Shellfish Immunology, 46(1), 94-106. https://doi.org/10.1016/j.fsi.2015.05.012.

Wendelboe, A. M. \& Raskob, G. E. (2016). Global Burden of Thrombosis: Epidemiologic Aspects. Circulation Research, 118(9), 1340-1347. https://doi.org/10.1161/ CIRCRESAHA.115.306841.

Zappelli, C., van der Zwaan, C., Thijssen-Timmer, D. C., Mertens, K. \& Meijer, A. B. (2012). Novel role for galectin-8 protein as mediator of coagulation factor $\mathrm{V}$ endocytosis by megakaryocytes. The Journal of Biological Chemistry, 287(11), 8327-8335. https://doi.org/10.1074/ jbc.M111.305151. 